
\section{(8} (9)

\title{
The astrophysical context of collision processes in meteorites
}

\author{
Yves Marrocchi ${ }^{1, *}$, Marco Delbo ${ }^{2} \&$ Matthieu Gounelle ${ }^{3}$ \\ ${ }^{1}$ Université de Lorraine, CNRS, Centre de Recherches Pétrographiques et Géochimiques \\ (CRPG), UMR 7358, Vandoeuvre les Nancy, F-54501, France \\ ${ }^{2}$ Université Côte d'Azur, Observatoire de la Côte d'Azur, CNRS, Laboratoire Lagrange, CS \\ 34229, 06304 Nice, France \\ ${ }^{3}$ IMPMC, Muséum national d'Histoire naturelle, Sorbonne Universités, CNRS, UMR 7590, \\ 57 rue Cuvier, 75005 Paris, France \\ *Corresponding author: yvesm@crpg.cnrs-nancy.fr
}

\section{Abstract}

Chondrites are leftover solids from the early evolution of the solar protoplanetary disk that never experienced melting since their formation. They comprise unequilibrated assemblages of low- and high-temperature components, including volatile-rich, fine-grained matrices, Fe-Ni metal, sulfides, refractory inclusions, and chondrules. Consequently, chondrites are commonly described as pristine, primitive, or primordial rocks of the solar system. However, impact-generated secondary features are abundant in chondrites, suggesting that collisions among early-formed planetesimals and their fragmentation and reassembly have been effective throughout the evolution of the solar system. In this report, we review evidence of the major role of impacts in generating the current mineralogical and petrographic characteristics of chondrites. We provide perspective to these meteoritic features by discussing recent analyses of large-scale structures of the main asteroid belt and remotesensing observations of asteroids. Observations at various spatial scales all attest that the 'primitive' materials formed during the evolution of the solar system have largely been 
32

33 that (i) chondrites (and some differentiated meteorites) should systematically be envisioned as

34 35 36 37 38 39 40 41 42 43 44 45 46 47 48 49

reprocessed, confirming previous studies that primitivity is relative, not absolute. This implies reprocessed and heterogeneous materials and (ii) brecciated meteorites should be considered the norm and unbrecciated meteorites the exception.

(1)

8 Keywords: chondrites, clasts, brecciation, asteroids, impacts 9 40 1 2 43 44 45 46 47 8 9 (1) 1 52 53 54 


\section{1- Introduction}

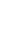

Meteorites are asteroidal (or rarely cometary; Gounelle et al. 2006) fragments recovered at the Earth's surface either promptly after their falls (e.g., Hiroi et al., 2001; Jenniskens et al. 2012; Marrocchi et al. 2020) or during field trips to cold/hot deserts (e.g., Bischoff and Geiger 1995; Evatt et al. 2020; Gattacceca et al. 2011; Harvey 2003; Schlüter et al. 2002). Meteorites' parent bodies are leftovers from the planetary formation processes that operated during the evolution of the solar system. Differentiated parent bodies recorded processes such as metal-silicate differentiation, early volcanism, granite-like magmatism and magma ocean episodes (e.g., Bischoff et al. 2014; Terada and Bischoff, 2009). In contrast, chondritic parent bodies did not experience melting and comprise high-temperature components formed in the solar protoplanetary disk (SPD) before planetesimal accretion, namely refractory inclusions (Ca-Al-rich inclusions, CAIs, and amoeboid olivine aggregates, AOAs; e.g., Marrocchi et al. 2019a) and chondrules (e.g., Connolly and Jones 2016). Chondrites thus represent invaluable witnesses of the SPD's evolution because they are made of components that formed at different times and locations in the SPD (Bollard et al. 2019; Connelly et al. 2012; Kawasaki et al. 2019; MacPherson et al. 2012; Pape et al. 2019; Petitat et al. 2011; Villeneuve et al. 2009).

However, most chondrites experienced subsequent thermal and hydrothermal alteration during asteroidal evolution, which blurred their initial textures, mineralogies, and chemical and isotopic compositions (Amsellem et al. 2020; Brearley 2006; Fujiya et al. 2015; Huss et al. 2006; Marrocchi et al. 2018a, 2021; Turner et al. 2021; Vacher et al. 2017, 2019a, 2019b, 2020). Based on these modifications, chondrites are assigned petrologic types on a scale from 1 to 6 , reflecting the progressive roles of low-temperature aqueous alteration (from type 3 to 1) and thermal metamorphism (from type 3 to 6; van Schmus and Wood, 1967). 
Type-3 chondrites thus correspond to materials that underwent minimal modifications after their agglomeration. Despite having suffered hydrothermal alteration of varying intensity, high-temperature components in type- 2 chondrites are thought to preserve evidence of the conditions of dust formation in the SPD (e.g., Chaumard et al. 2018; Kööp et al. 2016; Marrocchi et al. 2018b, 2019b; Piani et al., 2021). Consequently, these peculiar chondrites are commonly described as pristine, primitive, or primordial rocks of the solar system (Gounelle 2011; Libourel et al. 2017).

In addition to secondary alteration processes, hypervelocity impacts are of fundamental importance and played a key role in the early solar system and during its evolution (Bischoff et al., 2021; Raymond and Morbidelli 2020), yet have often been overlooked or treated in unrelated contexts (e.g., Lauretta and McSween 2006). The classical model of planetary formation involves three main steps, all entailing collisional processes. In the first, dust particles experience low-velocity collisions while migrating toward the disk's midplane, thus forming fractal aggregates that subsequently form pebbles through collisioninduced compaction (Blum and Wurm 2008; Johansen et al. 2007). The second step is characterized by the collision-induced coagulation of planetesimals, which forms massive planetary embryos and cores (Morbidelli et al. 2016). In the third step, gravitational instabilities induce collisions between planetary embryos, forming terrestrial planets on a time scale of $10^{7}-10^{8}$ years (Raymond and Morbidelli 2020; Raymond et al. 2009). Furthermore, the Moon, whose presence next to the Earth has shaped its evolution, is the result of a giant impact within the first 100 million years of the formation of CAIs (Canup 2012; Canup and Asphaug 2001). Fundamentally, all steps of planetary formation involve collisional processes, implying that they played a key role in shaping the present architecture of the solar system and that they are not an epiphenomenon that can be treated independently of other processes. 

driving the evolution of the asteroid belt, planetary and moon surfaces, and the formation and sustainability of Saturn's rings (Crida and Charnoz 2012). This is exemplified by the complex structures of boulders on near-Earth asteroids (101955) Bennu and (162173) Ryugu, as recently revealed by high-spatial-resolution images obtained by NASA's and JAXA's sample

109 return missions OSIRIS-REx and Hayabusa2, respectively (DellaGiustina et al. 2019;

110 Jaumann et al. 2019; Lauretta et al. 2019a, 2019b; Sugita et al. 2019). In particular, the 111 discovery of craters in the surfaces of Bennu's boulders revealed the effects of meteoroid 112 impacts on this asteroid, which occurred only in the last 1.75 Myr (Ballouz et al. 2020).

113 Furthermore, recent analyses of the main asteroid belt have revealed that only a very limited 114 number of the asteroids that we catalogue today have survived intact from primordial times 115 (Delbo et al. 2017), whereas the large majority of asteroids are fragments (Dermott et al. 2018) that experienced disruption of their parent bodies and re-accumulation (Michel 2001;

117 Michel and Richardson 2013; Michel et al. 2020). In this report, we thus aim to (i) detail the 118 astrophysical context of collisional processes in meteorites and (ii) put recent astrophysical 119 and planetological observations in a chondritic perspective.

\section{2- Meteoritic evidence of widespread collision processes in the asteroid belt}

Asteroidal collisions represent a major evolutionary process for solid bodies in the

124 solar system and have played a key role in establishing chondritic features, even in the 125 putative 'primitive' type-2 and -3 chondrites. This is attested by the ubiquitous occurrence of 126 brecciated textures in meteorites, with $\sim 30 \%$ of ordinary chondrites (OCs) and almost all CM 127 carbonaceous chondrites showing brecciation features (Fig. 1A; Bischoff et al. 1998; 2006; 128 Gattacceca et al. 2017; Lentfort et al., 2021; Suttle et al., 2021). Such characteristics are 
129 fundamental because (i) OCs are by far the most common meteorites falling on Earth ( 80\%)

130 and (ii) CMs represent 25\% of all carbonaceous chondrite falls (Gounelle et al. 2005). Even

131 rarer chondrite types like CI carbonaceous chondrites (Alfing et al. 2019), Rumuruti

132 chondrites (Bischoff et al. 2011), C1 chondrites (Bischoff et al., 2021), CB/CH chondrites

133 (Briani et al., 2009; Bonal et al., 2010), and enstatite chondrites (Fig. 1B; Piani et al., 2016,

134 2020) have brecciated textures, thus implying that all chondrite types have been reprocessed

135 after the accretion of their respective parent bodies.

136 Different types of brecciation are commonly observed among meteorites, revealing

137 that collision and re-agglomeration processes occurred at varying scales and intensities

138 (Bischoff et al. 2006; Rubin 1983). Monomict breccias are the most common and correspond

139 to meteorites formed by the in situ progressive fracturing and compaction of a single lithology

140 at a scale much smaller than their asteroidal parent bodies. Impact-melt breccias (Fig. 1B) are

141 rocks in which the matrix cementing the brecciated fragments crystallized from impact-

142 generated melts. Genomict breccias define meteorites containing fragments of the same

143 compositional group, but with different metamorphic or hydrothermal alteration histories

144 (Fig. 2; Gattacceca et al. 2017; Vacher et al. 2018; Verdier-Paoletti et al. 2019; Stöffler et al.,

145 2018); these textures reflect large-scale brecciation processes occurring within a given parent

146 body. In contrast, polymict breccias define meteorites composed of fragments originating

147 from different parent bodies (Fig. 3; Bischoff et al. 2006; Zolensky and Ivanov 2003); these

148 are of fundamental importance as they (i) record asteroidal mixing processes and (ii) contain

149 unique clasts not observed as individual samples (Patzek et al. 2018, 2020). The violent

150 evolution of the solar system is also evidenced by commonly observed petrofabrics and/or

151 flattened chondrules and refractory inclusions (Fig. 4; Gattacceca et al. 2005; Greashake

152 2014; Hanna et al. 2015; Rubin 2012; Vacher et al. 2018), which are generally interpreted to

153 result from impact-induced preferential orientations. 
155 by different lithologies than their respective host rocks (e.g., Bischoff et al. 2006; Bonal et al. 156 2010; Briani et al. 2009, 2012; Liu et al. 2020; Patzek et al. 2020; Zolensky et al. 1996) -

157 resulting from the low-velocity incorporation (i.e., $<1 \mathrm{~km} \mathrm{~s}^{-1}$; Briani et al. 2011) of 158 micrometeoroids by asteroidal parent bodies (Briani et al. 2011; Rubin and Bottke 2009).

159 Such clasts differ from polymict breccias because they could have been incorporated into

160 asteroids throughout the evolution of the solar system. The mixing of the host and xenoliths 161 probably at low-velocity is also attested by the lack of thermal metamorphism between the 162 clasts and their host meteorites (Fig. 6). Interestingly, the very few occurrences of CC clasts 163 in OC chondrites could result from the fragile nature of carbonaceous materials relative to

164 their OC counterparts. Taken together, brecciated textures, xenolith clasts, and petrofabrics 165 thus all attest to the violent evolution of the solar system, both during the first $~ 5-10$ Myr of 166 the SPD's evolution and continuing today.

Finally, the presence of high-pressure minerals in most meteorite groups is strong evidence of pervasive impacts on their parent bodies. Indeed, most of these minerals have been found within or adjacent to dark shock veins. Following the discovery of ringwoodite-

170 the spinel-structured polymorph of olivine - in Tenham, a large variety of shock-induced minerals have been discovered, including majorite, akimotoite (the ilmenite-structured polymorph of $\mathrm{MgSiO}_{3}$ ), and plagioclase with the hollandite structure (Sharp and DeCarli, 2006). At present, high-pressure minerals are used to constrain the pressures, temperatures,

174 and timescales of collision-induced shocks.

\section{3- The chaotic origin of the main asteroid belt}

The first step of asteroid formation occurred $\sim 4.56 \mathrm{Ga}$ when dust grains within the 
diameter. These were the first solid objects in the solar system whose internal strengths were

181 dominated by self-gravity (Johansen et al. 2007; Klahr and Schreiber 2020; Marcus 1967;

182 Morbidelli and Raymond 2016; Safronov and Zvjagina 1969). Although these objects

183 represent the main building blocks of terrestrial planets and the cores of giant planets, their

184 formation conditions and initial size distribution remain a matter of debate (Klahr and

185 Schreiber 2020). Classical models are based on the bottom-up progressive coagulation of

186 small solids into planets via merging collisions. However, during the disk's evolution, such

187 bottom-up planetesimal accretion is fundamentally limited by the 'meter-sized barrier': meter-

188 sized planetesimals will experience (i) rapid drift toward the central star and associated

189 evaporation and (ii) destruction during high-speed collisions (Wetherill 1990; Wurm et al.

190 2005). Recent models thus suggest that planetesimals were initially large (100-1,000 km) due

191 to high concentrations of small solid particles in turbulent structures of the SPD (Cuzzi et al.

192 2008; Johansen et al. 2007; Morbidelli et al. 2009). Such models are in agreement with

193 studies reconstructing the size distribution of planetesimals from analyses of the current

194 asteroid population (Bottke et al. 2005; Delbo et al. 2017, 2019; Morbidelli et al. 2009;

195 Tsirvoulis et al. 2018). However, most known asteroids are much smaller than planetesimals

196 (Masiero et al. 2014), indicating that they are remnants produced by the collisional

197 fragmentation of larger parent bodies, including planetesimals, throughout the history of the

198 solar system. An investigation of the dynamical behavior of asteroids reached the same

199 conclusion (Dermott et al. 2018). These results imply that (i) the current asteroid population is

200 the result of a dramatic collisional evolution that must also be reflected in the physical and

201 textural properties of meteorites and (ii) similar to meteorites, the current architecture of asteroid belt cannot be considered as a direct witness of the early solar system.

Most of planetesimal collisions have occurred in the main asteroid belt, between the

204 orbits of Mars and Jupiter (heliocentric distances of 2-3.2 AU), where most known asteroids 
are currently located. Contrary to common perceptions, the main belt is mostly empty, 206 containing only $5 \times 10^{-4}$ Earth masses (i.e., $\sim 4 \%$ of the mass of the Moon), with asteroids spread over an annular area more than three times as wide as the distance between Mercury and Mars. In addition, about half of the main-belt mass is contained in the four largest asteroids: Ceres, Vesta, Pallas, and Hygiea (DeMeo and Carry 2014). The main belt is 210 dynamically excited, with orbital eccentricities (e) up to 0.3 and inclinations $(i)$ of 0 to $>20^{\circ}$, and is characterized by the presence of spectroscopically distinct asteroids that, in general, correspond to distinct compositions (Fig. 7; Bus and Binzel 2002a, 2002b; DeMeo and Carry 2014): C-type asteroids are water-rich and genetically linked to carbonaceous chondrites, whereas S-type asteroids are dry and related to OCs. The compositions of asteroids vary as a function of their orbital semimajor axis (a): S-type asteroids are more abundant in the inner and central main belt $(2.1<a<2.7 \mathrm{AU})$ whereas C-type asteroids are more abundant in the outer regions $(2.7<a<3.2 \mathrm{AU})$.

Since the discovery of the first asteroids in the early nineteenth century, the main belt was long considered to be a 'living relic' of planetary formation. Canonical planetary formation theories postulated that (i) asteroids remained at the heliocentric distances at which they formed (as for planetesimals; Gradie and Tedesco 1982) and (ii) the compositional gradient of the main belt reflects that of the SPD from the inner border of the protoplanetary disk (a few tenths of an AU from the Sun) out to Jupiter (Raymond et al. 2006). However, these views began to shift in the mid-2000s. First, it was proposed that iron-meteorite parent bodies could have formed in the region of terrestrial planets before being scattered to the main belt by gravitational interactions with the growing terrestrial protoplanets (Bottke et al. 2006). Second, dynamic models suggested that the D-type asteroids observed today in the main belt are not indigenous, but instead correspond to trans-Neptunian objects that were scattered into the main belt by the giant planets during their instability phase (Fujiya et al. 2019; Levison et 
al. 2009; Marrocchi and Piani 2019; Morbidelli et al. 2010; Tsiganis et al. 2005). Following

231 these pioneering works, main-belt asteroid implantation models were advanced using new

232 dynamic models involving giant-planet formation and migration early in the solar system.

233 These models were developed to address two principle stumbling blocks of the classical

234 'static' models: they systematically produce (i) Mars analogs as massive as the Earth, rather

235 than about a tenth of the Earth's mass, and (ii) eccentricity and inclination distributions of

236 asteroids that do not match those of the current belt. Although important uncertainties remain,

237 the following three recent models (Fig. 7) have successfully reproduced these peculiar

238 characteristics and suggest that the entire main belt population of dynamically excited and

239 radially mixed S- and C-type asteroids are not indigenous, but were implanted in their current

240 locations between the terrestrial- and giant-planet regions.

242 (i) The "grand tack" model proposed that the inward and subsequent outward migrations of

243 Jupiter and Saturn in the protoplanetary disk truncated the disk at 1 AU, limiting the material 244 available for the formation of Mars (Fig. 7; Walsh et al. 2011). In this scenario, an initial 245 population of planetesimals was expelled from the main belt by the inward migration of 246 Jupiter, which plunged through the main belt. The main belt was subsequently repopulated by 247 planetesimals during the outward migration of Jupiter (Walsh et al. 2011). In this scenario, 248 planetesimals suffered mutual collisions at velocities $>10 \mathrm{~km} \mathrm{~s}^{-1}$ (Johnson et al. 2016).

250 (ii) The low-mass asteroid belt model preserves the concept of implanting planetesimals 251 into the main belt from the terrestrial- (S-types) and giant-planet regions (C-types; Raymond 252 and Izidoro 2017a, 2017b). However, contrary to other models such as the grand tack model, 253 these authors showed that the main belt could have initially had a low mass (or could have 254 even been empty) and that large-scale migrations of the giant planets are not required to 
populate it with the current mixture of asteroid types (Fig. 7). In this model, C- and S-type 256 asteroids were implanted not only from different sources but also at different times: C-type 257 planetesimals that formed in the giant-planet region were scattered inward during the gas phase of the protoplanetary disk (i.e., within a few million years after the formation of CAIs; Raymond and Izidoro 2017a, 2017b), whereas S-type asteroids were implanted from the 260 terrestrial-planet region into the main belt after the gas in the protoplanetary disk had 261 dissipated (i.e., later than $\sim 10 \mathrm{Myr}$ after the formation of CAIs). The implantation of S-type asteroids was slower and more prolonged than that of C-type asteroids, possibly lasting 100 Myr (Raymond and Izidoro 2017b) because, in this model, S-type planetesimals must have been scattered via multiple close encounters with planetary embryos. In such models, Mars' small mass results from a primordial mass deficit between the present-day orbits of Earth and Jupiter (Hansen 2009).

(iii) The early instability model recently provided an alternative view in which the giant planets did not undergo large migrations during the gas phase (Fig. 7; Clement et al. 2018, 2019a, 2019b). In this model, as in the canonical scenario, a sea of planetesimals spanning the inner rim of the protoplanetary disk (out to Jupiter) was perturbed by an early orbital instability of the giant planets around $10 \mathrm{Myr}$ after gas dispersal, which triggered the formation of the terrestrial planets. This instability also removed significant mass from the main-belt region and broadened the ranges of the eccentricities and inclinations of main-belt 275 planetesimals to the current values, thereby starting the violent collisional evolution of these 276 bodies.

Although the current orbital structure (semi-major axis, eccentricity, inclination) of the asteroid belt is consistent with an initially uniform distribution of asteroids (Minton and 
Malhotra 2009), its self-excitation requires several orders of magnitude more mass than is 281 present today (e.g., O'Brien and Sykes 2011). This implies that the belt experienced violent processes that considerably modified its initial structure. Whatever the process that dominantly shaped the asteroid belt, collisional processes were important and common during

284 the early evolution of the solar system. Depending on the scenario invoked, the main belt 285 reached its current dynamic configuration after tens to hundreds of millions of years (e.g., 286 after giant-planet instability). Since then, collisions have continued to occur with typical 287 impact velocities between asteroids of $\sim 5.3 \mathrm{~km} \mathrm{~s}^{-1}$ (Bottke et al. 2005 and references therein), 288 although the impact velocities define a broad distribution and collisions at higher-than289 average speeds are not uncommon, particularly for objects with high $i$ and/or $e$ values. One 290 such case is the third-largest asteroid (2) Pallas $\left(i=34.8^{\circ}, e=0.23\right)$, for which impact 291 velocities are estimated to be $\sim 12 \mathrm{~km} \mathrm{~s}^{-1}$ on average (Marsset et al. 2020). Collisions at such 292 speeds can yield temperatures high enough to induce the dehydration and thermal 293 metamorphism of asteroidal materials, which should be observable in the meteorites that we 294 may receive from these bodies (Amsellem et al. 2020 and references therein). This collisional 295 evolution is responsible for the formation of the families of asteroid fragments (Nesvorny et 296 al. 2015) that we observe in the main belt. The effects of these collisions, which can lead to 297 the mixing of asteroidal materials of different compositions, is also visible in some physical 298 properties of asteroids, as it is detailed in the next section.

\section{4- Evidence for collisional processes from in-situ exploration of asteroids} growing evidence of the effects of impacts and the resulting material mixing produced by the

304 infall of exogenic impacting objects. 
The first evidence of the violent collisional evolution of asteroids is the presence of craters on their surfaces. This has been clear since NASA's Galileo mission, the first spacecraft to fly by an asteroid, which imaged (951) Gaspra in 1991 (Veverka et al. 1994) and showed that its surface is covered by impact craters (Stooke 1996). Since then, impact craters appear to be ubiquitous structures as they have been observed on all imaged asteroids. These craters range in size from the $\sim 500$-km-diameter Rheasilvia basin near the south pole of (4)

311 Vesta (Schenk et al. 2012; Thomas et al. 1997) to decimeter-scale craters in boulders on the 312 surface of the 500-m-diameter near-Earth asteroid (101955) Bennu (Ballouz et al. 2020). On 313 Vesta, the deposition of the so-called 'dark material' that is often associated with craters has 314 been attributed to impacts by carbonaceous asteroids (McCord et al. 2012). This dark material 315 is then (i) diffused throughout the regolith of the surface of Vesta over time (McCord et al. 2012) and (ii) compressed by subsequent impacts on the surface and cemented together with original Vestan material (brighter in color) into breccia-like rocks. This process is in good agreement with the common presence of clasts of carbonaceous chondritic materials within 319 Howardites (e.g., Fig. 5; Buchanan et al., 1993; Liu et al. 2020; McSween et al. 2011; van 320 Drongelen et al., 2016), regolith breccia meteorites belonging to the HED group and 321 originating from the surface of Vesta.

Because Vesta is a differentiated asteroid, it has been proposed that impacts could excavate its basaltic crust, revealing its underlying olivine mantle. However, very little olivine 324 has been detected at Vesta's surface, even in and around the giant impact basins Rheasilvia 325 and Venenia (Ammannito et al. 2013; De Sanctis et al. 2012). Therefore, the rare occurrences 326 of olivine signatures on Vesta's surface may rather correspond to material implanted by 327 olivine-rich impactors (Turrini et al. 2016). Indeed, laboratory impact experiments performed 328 at velocities similar to the average impact velocity on Vesta demonstrated that impactor 329 material could be preserved at Vesta's surface (Daly and Schultz 2016). Although the 
survivability and implantation of impactor material is a function of impact velocity,

331 laboratory hypervelocity impact experiments on porous and non-porous targets showed that

332 impact-generated material implantation is a general process occurring throughout the solar system (Avdellidou et al. 2016, 2017). This implies that the mixing of asteroidal materials was possible not only during the early gas phase of the SPD when impact velocities were lower, but throughout the history of the solar system. Interestingly, high-speed collisions (i.e., $12 \mathrm{~km} \mathrm{~s}^{-1}$; Marsset et al. 2020) can also yield temperatures high enough to induce the dehydration and thermal metamorphism of asteroidal materials (Amsellem et al. 2020 and references therein).

Very recent observations at $\mathrm{cm}$-scale spatial resolution of the surfaces of the near-

340 Earth asteroids (101955) Bennu and (162173) Ryugu by NASA's OSIRIS-REx (Lauretta et al. 2019a, 2019b) and JAXA's Hayabusa2 (Okada et al. 2020; Tatsumi et al. 2021) sample return missions, respectively, provide a wealth of indications about the mixing of asteroidal materials and the presence of brecciated rocks on these asteroids. OSIRIS-REx spectroscopic

344 and spectrophotometric observations revealed the presence of bright, pyroxene-rich boulders of Vesta-like composition on the dark, carbonaceous surface of Bennu (DellaGiustina et al. 2020). This suggests that some asteroids of the Vesta collisional family impacted Bennu, or 347 more likely its parent body, mixing the two materials (DellaGiustina et al. 2020). This is also 348 attested by the peculiar textures of some of Bennu's pyroxene-rich, hummocky boulders, 349 which show potential layering or brecciation (DellaGiustina et al. 2020). Furthermore, two 350 bright, pyroxene-bearing clasts appear embedded within a large, partially buried boulder, the 351 latter having an albedo similar to the average of Bennu (DellaGiustina et al. 2020). 352 Interestingly, the Hayabusa2 orbiter also detected bright material on the dark surface of 353 Ryugu (Tatsumi et al. 2021), thus suggesting that brecciation is a common process during 354 asteroidal evolution. 
In contrast to Bennu, the spectral signatures of Ryugu indicate two distinct units of bright material. One appears to be more compatible with an S-type asteroidal composition (rather than a pyroxene-rich, Vesta-like nature; Tatsumi et al. 2021) that could have been delivered by an impact (or series of impacts) on Ryugu's parent body. The second unit has spectral features similar to the bulk of Ryugu, but with a less pronounced upturn at ultraviolet

360 frequencies compared to the Ryugu average (Tatsumi et al. 2021). This unit may represent a 361 more thermally metamorphosed material compared to that covering most of the surface of the 362 asteroid (Tatsumi et al. 2021). It thus appears that Ryugu's surface comprises materials originating from various depths within its parent body. Such diverse materials may also be present on other similar asteroids, compacted together by small-scale impacts to form breccias. boulders with albedo and/or color heterogeneities and (ii) non-fractured boulders containing meter-sized clasts (Lauretta et al. 2019a). Such assemblages have been interpreted as impact breccias (e.g., see Fig. 1b of Walsh et al. 2019). However, the collisional processes capable of producing breccia-like boulders several meters in size and containing meter-scale clasts are 371 probably too energetic to be withstood by Bennu itself (Bischoff et al. 2006). Hence, these boulders probably formed on Bennu's parent body and may record (i) the parent body's accretion in the SPD (Walsh et al. 2019), (ii) compaction of regolith blocks on the parent 374 body, or (iii) collisional events that fragmented the parent body to form Bennu. The surfaces 375 of some boulders larger than ten meters in size also appear to be "littered" with brighter 376 centimetric rocks that, in some cases, appear to be cemented together (Bennett et al. 2021). 377 Although the origin of this material is unclear, it may result from (i) impacts on the boulders 378 themselves (Bennett et al. 2021) or (ii) particles falling back onto the asteroid after particle379 ejection events, such as were observed on Bennu (Lauretta et al., 2019b). 
To summarize, the presence of clasts and exogenic components in Bennu and Ryugu

381 imply that material-mixing via impacts is a common process on these bodies, and likely 382 throughout the solar system. These observations also give context to what was once a 383 breakthrough in asteroid and meteoritic science, namely the discovery of distinct meteorite 384 classes within the strewn field of the Almahata-Sitta meteorites, which were produced by the 385 atmospheric breakup of asteroid $2008 \mathrm{TC}_{3}$ (Bischoff et al. 2010; Goodrich et al., 2015, 2019;

386 Horstmann and Bischoff, 2014; Jenniskens et al. 2009). Before the Almahata-Sitta recovery, 387 the canonical concept was that one class of meteorite originates from one class of asteroid. 388 These recent spacecraft observations have thus demonstrated that $2008 \mathrm{TC}_{3}$ is perhaps not an 389 exception and that individual asteroids can host materials corresponding to diverse meteorite 390 classes.

\section{5- Implications and perspectives}

Brecciated textures, xenolith clasts, and petrofabrics in meteorites attest to the chaotic evolution of the solar system, both during the first 5-10 Myr of the evolution of the SPD and continuing until today. Given our knowledge of the structure of the asteroid belt and our theoretical understanding of the evolution of the solar system, this testifies that so-called primitive materials formed during the evolution of the SPD have been largely reprocessed, implying that primitivity is relative, not absolute (Bischoff et al. 2019; Gounelle 2011; Libourel et al. 2017). This is not greatly surprising as the spectra of $\sim 20 \%$ of nearby solartype stars of various ages show infrared peaks related to the presence of debris disks, lowmass (i.e., less than the mass of the Earth), low-density, dust-dominated disks that are continuously replenished by collisions between large planetesimals (Hughes et al. 2018).

404 Debris disks are analogous to our solar system's Kuiper Belt and zodiacal light and are 
fundamentally different from protoplanetary disks, which are dominated by agglomeration 406 processes whereas debris disks are destruction-dominated. This implies that early-formed 407 planetesimals (i) were largely reprocessed during the evolution of the solar system and (ii) experienced fragmentation and subsequent reassembly as asteroids observed today. Indeed, 409 the astronomical survey of the asteroid belt suggests that only a few hundreds of asteroids 410 have remained unchanged since their formation (Delbo et al. 2017), clearly indicating that 411 solar system materials have been largely reprocessed via impacts. In addition, planetesimals 412 that accreted during the two million years following CAI formation likely experienced 413 melting due to ${ }^{26} \mathrm{Al}$ decay (Bizzarro et al. 2005; Hevey and Sanders 2006; Sahijpal et al. 414 2007), which, at least partially (i.e., primitive achondrites), erased their primordial features. The complex evolution of asteroids is an ongoing process; recent reports suggest that 416 impact-generated fluid circulations, probably triggered by hypervelocity impacts, have occurred within the past few hundreds of thousands of years (Amsellem et al. 2020; Turner et al. 2021). This is also highlighted by the ubiquitous presence of xenolith clasts in chondrites and achondrites (Bischoff et al. 2006; Bonal et al. 2010; Briani et al. 2009, 2012; Cohen et al., 2004; Liu et al. 2020; Patzek et al. 2018, 2020; Zolensky et al. 1996), which may have been incorporated into their current bodies by implantation processes and/or regolith gardening throughout the evolution of the solar system. Therefore, 'primitive' samples are probably extremely rare, and the concept of primitivity should only be used to relatively describe two or several objects, and always in terms of processes.

A direct consequence is the limitation of the so-called bulk composition of meteorites, as -depending on the scale sampled- the average composition could have been modified by various processes operating all along the solar system evolution. For example, although CI chondrites are commonly used as a reference for chemical normalization (Lodders 2003), CIs show brecciated structures and are significantly heterogeneous at a scale of just $\sim 100 \mathrm{mg}$ 
430 (Morlok et al. 2006) or up to $1 \mathrm{~g}$ for elements sensitive to fluid circulation (Barrat et al. 2012).

431 Therefore, virtually all chondrites (and some differentiated meteorites) should be viewed as 432 reprocessed, heterogeneous materials, and analyses of their bulk chemical and isotopic 433 compositions can only provide usable results when specifying the sampling scale. Taken 434 together, all these observations suggest the need for a new perspective on meteorites, 435 considering (i) highly brecciated meteorites (i.e., Kaidun and Almahata Sitta, typically viewed 436 as anomalies) as the norm and (ii) monomict breccias as the exception. In addition, the numerous outstanding questions regarding the origin, distribution, and evolution of xenolith clasts require (i) extensive mineralogical, petrographic, and isotopic characterizations of xenolith clasts (especially in OCs, for which only a limited number have been described), (ii) an understanding of the dynamics and chronology of their incorporation into their host bodies, and (iii) further development of the physics of small-scale implantation processes.

\section{Acknowledgments}

We thank Laurette Piani and Maximilien J. Verdier-Paoletti for providing chondrite pictures. This work is the result of intense scientific discussions during the February 2020 workshop "First solids and planetesimals: formation conditions and evolution" hosted in les Houches (France) and organized by the Programme National de Planétologie (PNP). Nan Liu and Markus Patzek are warmly thanked for positive and constructive reviews and we thank 


\section{Figure captions}

Fig. 1: A) Reflected-light photograph of a piece of the Boriskino CM chondrite, courtesy of

L. D. Bayle. Diverse lithologies, characterized by different shades of gray, are easily

distinguished. B) Reflected-light photograph of the enstatite chondrite Abee showing impactgenerated veins (Bischoff et al., 2006; Rubin, 2015). Courtesy of Laurette Piani.

Fig. 2: Back-scattered electron image of the brecciated Cold Bokkeveld CM chondrite. Individual fragments (some are traced by red dashed lines) are variable in composition, as indicated by their different shades of gray.

Fig. 3: Energy-dispersive X-ray elemental map of the $\mathrm{Mg}, \mathrm{Al}, \mathrm{Si}, \mathrm{Ca}$, and $\mathrm{Fe}$ distributions in the polymict breccia Kaidun, revealing its complex nature.

Fig. 4: Back-scattered electron image of CV3 Efremovka, showing a high degree of chondrule flattening induced by hypervelocity impacts. A fine-grained clast is also observed 471 in this section.

Fig. 5: Back-scattered electron images of xenolith clasts (central in each image) observed in (A) CB Isheyevo and (B) the Kapoeta Howardite.

Fig. 6: A) Back scattered electron image of the Paris chondrites showing the presence of

477 light-colored clasts (red boxes). B) and C) Back scattered electron images of the contacts 478 between the clasts and the host chondrites. No specific sign of thermal metamorphism is 479 observed. 
480 Fig. 7: Schematic comparison of the three global models currently proposed for explaining 481 the peculiar structure of the main asteroid belt and the solar system formation (described in 482 section 4$)$.

483

484

485

486

487

488

489

490

491

492

493

494

495

496

497

498

499

500

501

502

503

504 
References

Alfing J., Patzek M., and Bischoff A. 2019. Modal abundances of coarse-grained (>5 $\mu \mathrm{m}$ ) components within CI-chondrites and their individual clasts - Mixing of various lithologies on the CI parent body(ies). Geochemistry 79:125532.

Ammannito E. et al. 2013. Olivine in an unexpected location on Vesta's surface. Nature 504:122-125.

Amsellem E., Moynier F., Mahan B., and Beck P. 2020. Timing of thermal metamorphism in CM chondrites: Implications for Ryugu and Bennu future sample return. Icarus 339:113593.

Avdellidou C., Price M. C., Delbo M., Ioannidis P., and Cole M. J. 2016. Survival of the impactor during hypervelocity collisions - I. An analogue for low porosity targets. Monthly Notices of the Royal Astronomical Society 456:2957-2965.

Avdellidou C., Price M. C., Delbo M., and Cole M. J. 2017. Survival of the impactor during hypervelocity collisions - II. An analogue for high-porosity targets. Monthly Notices of the Royal Astronomical Society 464:734-738.

Ballouz R.-L. et al. 2020. Bennu's near-Earth lifetime of 1.75 million years inferred from craters on its boulders. Nature 587:205-209.

Barrat J. A., Zanda B., Moynier F., Bollinger C., Liorzou C., and Bayon G. 2012. Geochemistry of $\mathrm{CI}$ chondrites: Major and trace elements, and $\mathrm{Cu}$ and $\mathrm{Zn}$ Isotopes. Geochimica et Cosmochimica Acta 83:79-92.

Bennett C. A. et al. 2021. A high-resolution global basemap of (101955) Bennu. Icarus $357: 113690$.

Bischoff A. and Geiger T. 1985. Meteorites from the Sahara: Find locations, shock classification, degree of weathering, and pairing. Meteoritics 30, 113-122.

Bischoff A., Scott E. R. D., Metzler K., and Goodrich C. A. 2006. Nature and origins of meteoritic breccias. In Meteorites and the early solar system II, edited by Lauretta D. S. and McSween H. Y. Tucson, Arizona: The University of Arizona Press. pp. 679712.

Bischoff A., Horstmann M., Pack A., Laubenstein M., and Haberer S. 2010. Asteroid 2008 TC3-Almahata Sitta: A spectacular breccia containing many different ureilitic and chondritic lithologies: Almahata Sitta-A spectacular breccia. Meteoritics \& Planetary Science 45:1638-1656.

Bischoff A., Vogel N., and Roszjar J. 2011. The Rumuruti chondrite group. Geochemistry 71:101-133.

Bischoff A., Horstmann M., Barrat J.-A., Chaussidon M., Pack A., Herwartz D., Ward D., Vollmer C., and Decker S. 2014. Trachyandesitic volcanism in the early Solar System. Proceedings of the National Academy of Sciences 111:12689-12692. 
Bischoff A., Schleiting M., Wieler R., and Patzek M. 2018. Brecciation among 2280 ordinary chondrites - constraints on the evolution of their parent bodies. Geochimica et Cosmochimica Acta 238, 516-541.

Bischoff A., Schleiting M., and Patzek M. 2019. Shock stage distribution of 2280 ordinary chondrites - Can bulk chondrites with a shock stage of S6 exist as individual rocks? Meteoritics \& Planetary Science 54:2189-2202.

Bischoff A. et al. 2021. The old, unique C1 chondrite Flensburg - Insight into the first processes of aqueous alteration, brecciation, and the diversity of water-bearing parent bodies and lithologies. Geochimica et Cosmochimica Acta 293:142-186.

Bizzarro M., Baker J. A., Haack H., and Lundgaard K. L. 2005. Rapid Timescales for Accretion and Melting of Differentiated Planetesimals Inferred from 26Al-26Mg Chronometry. ApJ 632:L41-L44.

Blum J., and Wurm G. 2008. The Growth Mechanisms of Macroscopic Bodies in Protoplanetary Disks. Annual Review of Astronomy and Astrophysics 46:21-56.

Bollard J. et al. 2019. Combined U-corrected $\mathrm{Pb}-\mathrm{Pb}$ dating and 26Al-26Mg systematics of individual chondrules - Evidence for a reduced initial abundance of $26 \mathrm{Al}$ amongst inner Solar System chondrules. Geochimica et Cosmochimica Acta 260:62-83.

Bonal L., Huss G. R., Krot A. N., and Nagashima K. 2010. Chondritic lithic clasts in the $\mathrm{CB} / \mathrm{CH}$-like meteorite Isheyevo: Fragments of previously unsampled parent bodies. Geochimica et Cosmochimica Acta 74:2500-2522.

Bottke W. F., Nesvorný D., Grimm R. E., Morbidelli A., and O’Brien D. P. 2006. Iron meteorites as remnants of planetesimals formed in the terrestrial planet region. Nature 439:821-824.

Bottke W.F., Durda D., Nesvorný D., Jedicke R., Morbidelli A., Vokrouhlický D., and Levison H. 2005. The fossilized size distribution of the main asteroid belt. Icarus 175:111-140.

Brearley A. J. 2006. The action of water. In Meteorites and the early solar system II, edited by Lauretta D. S. and McSween H. Y. Tucson, Arizona: The University of Arizona Press. pp. 587-624.

Briani G., Gounelle M., Marrocchi Y., Mostefaoui S., Leroux H., Quirico E., and Meibom A. 2009. Pristine extraterrestrial material with unprecedented nitrogen isotopic variation. Proceedings of the National Academy of Sciences of the United States of America 106:10522-10527.

Briani G., Morbidelli A., Gounelle M., and Nesvorný D. 2011. Evidence for an asteroidcomet continuum from simulations of carbonaceous microxenolith dynamical evolution: Carbonaceous microxenoliths and the asteroid-comet continuum. Meteoritics \& Planetary Science 46:1863-1877.

Briani G., Gounelle M., Bourot-Denise M., and Zolensky M. E. 2012. Xenoliths and microxenoliths in H chondrites: Sampling the zodiacal cloud in the asteroid Main Belt. Meteoritics \& Planetary Science 47:880-902. 
Buchanan P. C., Zolensky M. E. and Reid A. M. 1993. Carbonaceous chondrite clasts in the howardites Bholghati and EET87513. Meteoritics \& Planetary Science 28: 659-669.

Bus S.J., and Binzel R.P. 2002a. Phase II of the Small Main-Belt Asteroid Spectroscopic Survey A Feature-Based Taxonomy. Icarus 158:146-177.

Bus S.J., and Binzel R.P. 2002b. Phase II of the Small Main-Belt Asteroid Spectroscopic Survey The Observations. Icarus 158:106-145.

Canup R. M., and Asphaug E. 2001. Origin of the Moon in a giant impact near the end of the Earth's formation. Nature 412:708-712.

Canup R. M. 2012. Forming a Moon with an Earth-like Composition via a Giant Impact. Science 338:1052-1055.

Chaumard N. 1, Defouilloy C., and Kita N. T. 2018. Oxygen isotope systematics of chondrules in the Murchison CM2 chondrite and implications for the CO-CM relationship. Geochimica et Cosmochimica Acta 228:220-242.

Clement M. S., Kaib N. A., Raymond S. N., and Walsh K. J. 2018. Mars' growth stunted by an early giant planet instability. Icarus 311:340-356.

Clement M. S., Raymond S. N., and Kaib N. A. 2019a. Excitation and Depletion of the Asteroid Belt in the Early Instability Scenario. The Astronomical Journal 157:38.

Clement M. S., Kaib N. A., Raymond S. N., Chambers J. E., and Walsh K. J. 2019b. The early instability scenario: Terrestrial planet formation during the giant planet instability, and the effect of collisional fragmentation. Icarus 321:778-790.

Cohen B. A., Goodrich C. A., and Keil K. 2004. Feldspathic clast populations in polymict ureilites: Stalking the missing basalts from the ureilite parent body. Geochimica et Cosmochimica Acta 68:4249-4266.

Connelly J. N., Bizzarro M., Krot A. N., and Nordlund A. 2012. The absolute chronology and thermal processing of solids in the solar protoplanetary disk. Science 338:651-655.

Connolly H. C., and Jones R. H. 2016. Chondrules: The canonical and noncanonical views. Journal of Geophysical Research: Planets 121:1885-1899.

Crida A., and Charnoz S. 2012. Formation of Regular Satellites from Ancient Massive Rings in the Solar System. Science 338:1196-1199.

Cuzzi J. N., Hogan R. C., and Shariff K. 2008. Toward planetesimals: Dense chondrule clumps in the protoplanetary nebula. The Astrophysical Journal 687:1432-1447.

Daly R. T., and Schultz P. H. 2016. Delivering a projectile component to the vestan regolith. Icarus 264:9-19.

De Sanctis M. C. et al. 2012. Spectroscopic Characterization of Mineralogy and Its Diversity Across Vesta. Science 336:697-700. 
Delbo M., Walsh K., Bolin B., Avdellidou C., and Morbidelli A. 2017. Identification of a primordial asteroid family constrains the original planetesimal population. Science 357:1026-1029.

Delbo M., Avdellidou C., and Morbidelli A. 2019. Ancient and primordial collisional families as the main sources of X-type asteroids of the inner main belt. Astronomy \& Astrophysics 624:A69.

DellaGiustina D. N. et al. 2019. Properties of rubble-pile asteroid (101955) Bennu from OSIRIS-REx imaging and thermal analysis. Nature Astronomy 3:341-351.

DellaGiustina D. N. et al. 2020. Variations in color and reflectance on the surface of asteroid (101955) Bennu. Science 370:eabc3660.

DeMeo F. E., and Carry B. 2014. Solar System evolution from compositional mapping of the asteroid belt. Nature 505:629-634.

Dermott S. F., Christou A. A., Li D., Kehoe Thomas. J. J., and Robinson J. M. 2018. The common origin of family and non-family asteroids. Nature Astronomy 2:549-554.

Evatt G. W., Smedley A. R. D., Joy K. H., Hunter L., Tey W. H., Abrahams I. D., and Gerrish L. 2020. The spatial flux of Earth's meteorite falls found via Antarctic data. Geology 48:683-687.

Fujiya W., Sugiura N., Marrocchi Y., Takahata N., Hoppe P., Shirai K., Sano Y., and Hiyagon H. 2015. Comprehensive study of carbon and oxygen isotopic compositions, trace element abundances, and cathodoluminescence intensities of calcite in the Murchison CM chondrite. Geochim. Cosmochim. Acta 161:101-117.

Fujiya W., Hoppe P., Ushikubo T., Fukuda K., Lindgren P., Lee M. R., Koike M., Shirai K., and Sano Y. 2019. Migration of D-type asteroids from the outer Solar System inferred from carbonate in meteorites. Nature Astronomy 3:910-915.

Gattacceca J., Rochette P., Denise M., Consolmagno G., and Folco L. 2005. An impact origin for the foliation of chondrites. Earth and Planetary Science Letters 234:351-368.

Gattacceca J. et al. 2011. The densest meteorite collection area in hot deserts: The San Juan meteorite field (Atacama Desert, Chile). Meteoritics \& Planetary Science 46:12761287.

Gattacceca J., Krzesińska A. M., Marrocchi Y., Meier M. M. M., Bourot-Denise M., and Lenssen R. 2017. Young asteroid mixing revealed in ordinary chondrites: The case of NWA 5764, a polymict LL breccia with L clasts. Meteoritics \& Planetary Science. 44:431-16.

Goodrich C. A., Hartmann W. K., O’Brien D. P., Weidenschilling S. J., Wilson L., Michel P., and Jutzi M. 2015. Origin and history of ureilitic material in the solar system: The view from asteroid $2008 \mathrm{TC}_{3}$ and the Almahata Sitta meteorite. Meteoritics \& Planetary Science 50:782-809. 
Goodrich C. A. et al. 2019. The first samples from Almahata Sitta showing contacts between ureilitic and chondritic lithologies: Implications for the structure and composition of asteroid $2008 \mathrm{TC}_{3}$. Meteoritics \& Planetary Science 54:2769-2813.

Gounelle M., Engrand C., Alard O., Bland P. A., Zolensky M. E., RUSSELL S. S., and Duprat J. 2005. Hydrogen isotopic composition of water from fossil micrometeorites in howardites. Geochimica et Cosmochimica Acta 69:3431-3443.

Gounelle M., Spurný P., and Bland P. A. 2006. The orbit and atmospheric trajectory of the Orgueil meteorite from historical records. Meteoritics \& Planetary Science 41:135150.

Gounelle M. 2011. The Asteroid-Comet Continuum: In Search of Lost Primitivity. Elements 7:29-34.

Gradie J., and Tedesco E. 1982. Compositional Structure of the Asteroid Belt. Science 216:1405-1407.

Greshake A. 2014. A strongly hydrated microclast in the Rumuruti chondrite NWA 6828: Implications for the distribution of hydrous material in the solar system. Meteoritics \& Planetary Science 49:824-841.

Hanna R. D., Ketcham R. A., Zolensky M., and Behr W. M. 2015. Impact-induced brittle deformation, porosity loss, and aqueous alteration in the Murchison CM chondrite. Geochimica et Cosmochimica Acta 171:256-282.

Hansen B. M. S. 2009. Formation of the terrestrial planets from a narrow annulus. Astrophysical journal 703:1131-1140.

Harvey R. 2003. The Origin and Significance of Antarctic Meteorites. Geochemistry 63:93147.

Hevey P. J., and Sanders I. S. 2006. A model for planetesimal meltdown by ${ }^{26} \mathrm{Al}$ and its implications for meteorite parent bodies. Meteoritics \& Planetary Science 41:95-106.

Hiroi T., Zolensky M.E., and Pieters C.M. 2001. The Tagish Lake Meteorite: A possible Sample from a D-Type Asteroid. Science 293:2234-2236.

Horstmann M. and Bischoff A. (2014). The Almahata Sitta polymict breccia and the late accretion of Asteroid 2008 TC3 - Invited Review. Chemie der Erde - Geochemistry $74,149-184$.

Hughes A. M., Duchene G., and Matthews B. 2018. Debris Disks: Structure, Composition, and Variability. Annual Review of Astronomy and Astrophysics 56:541-591.

Huss G. R., Rubin A. E., and Grossman J. N. 2006. Thermal Metamorphism in Chondrites. In Meteorites and the early solar system II, edited by Lauretta D. S. and McSween H. Y. Tucson, Arizona: The University of Arizona Press, pp. 567-586.

Jaumann R. et al. 2019. Images from the surface of asteroid Ryugu show rocks similar to carbonaceous chondrite meteorites. Science 365:817-820. 
Jenniskens P. et al. 2009. The impact and recovery of asteroid 2008 TC3. Nature 458:485488.

Jenniskens P. et al. 2012. Radar-Enabled Recovery of the Sutter's Mill Meteorite, a Carbonaceous Chondrite Regolith Breccia. Science 338:1583-1587.

Johansen A., Oishi J. S., Low M.-M. M., Klahr H., Henning T., and Youdin A. 2007. Rapid planetesimal formation in turbulent circumstellar disks. Nature 448:1022-1025.

Johnson B. C., Walsh K. J., Minton D. A., Krot A. N., and Levison H. F. 2016. Timing of the formation and migration of giant planets as constrained by CB chondrites. Science Advances 2:e1601658.

Kawasaki N., Park C., Sakamoto N., Park S. Y., Kim H. N., Kuroda M., and Yurimoto H. 2019. Variations in initial 26Al/27Al ratios among fluffy Type A Ca-Al-rich inclusions from reduced CV chondrites. Earth and Planetary Science Letters 511:2535.

Klahr H., and Schreiber A. 2020. Turbulence Sets the Length Scale for Planetesimal Formation: Local 2D Simulations of Streaming Instability and Planetesimal Formation. The Astrophysical Journal 901:54.

Kööp L., Davis A. M., Nakashima D., Park C., Krot A. N., Nagashima K., Tenner T. J., Heck P. R., and Kita N. T. 2016. A link between oxygen, calcium and titanium isotopes in 26Al-depleted hibonite-rich CAIs from Murchison and implications for the heterogeneity of dust reservoirs in the solar nebula. Geochimica et Cosmochimica Acta 1-43.

Lauretta D.S., and McSween H.Y. 2006. Meteorites and the early solar system II. Tucson, Arizona: The University of Arizona Press, 943 p.

Lauretta D. S. et al. 2019a. The unexpected surface of asteroid (101955) Bennu. Nature 1-16.

Lauretta D. S. et al. 2019b. Episodes of particle ejection from the surface of the active asteroid (101955) Bennu. Science 366:eaay3544.

Lentfort S., Bischoff A., Ebert S., and Patzek M. 2021: Classification of CM chondrite breccias - implications for the evaluation of samples from the OSIRIS-REx and Hayabusa 2 missions. Meteoritics \& Planetary Science 56, 127-147.

Levison H. F., Bottke W. F., Gounelle M., and Morbidelli A. 2009. Contamination of the asteroid belt by primordial trans-Neptunian objects. Nature 460:364-366.

Libourel G., Michel P., Delbo M., Ganino C., Recio-Blanco A., de Laverny P., Zolensky M. E., and Krot A. N. 2017. Search for primitive matter in the Solar System. Icarus 282:375-379.

Liu N., Ogliore R. C., and Vacher L. G. 2020. NanoSIMS isotopic investigation of xenolithic carbonaceous clasts from the kapoeta howardite. Geochimica et Cosmochimica Acta 283:243-264. 
Lodders K. 2003. Solar system abundances and condensation temperatures of the elements. The Astrophysical Journal 591:1220-1247.

MacPherson G. J., Kita N. T., Ushikubo T., Bullock E. S., and Davis A. M. 2012. Wellresolved variations in the formation ages for $\mathrm{Ca}-\mathrm{Al}$-rich inclusions in the early Solar System. Earth and Planetary Science Letters 331-332:43-54.

Marcus A. H. 1967. Formation of the planets by accretion of planetesimals: Some statistical problems. Icarus 7:283-296.

Marrocchi Y., Bekaert D. V., and Piani L. 2018a. Origin and abundance of water in carbonaceous asteroids. Earth and Planetary Science Letters 482:23-32.

Marrocchi Y., Villeneuve J., Batanova V., Piani L., and Jacquet E. 2018b. Oxygen isotopic diversity of chondrule precursors and the nebular origin of chondrules. Earth and Planetary Science Letters 496:132-141.

Marrocchi Y., Villeneuve J., Jacquet E., Piralla M., and Chaussidon M. 2019a. Rapid condensation of the first Solar System solids. Proceedings of the National Academy of Sciences of the United States of America 116:23461-23466.

Marrocchi Y., Euverte R., Villeneuve J., Batanova V., Welsch B., Ferrière L., and Jacquet E. $2019 \mathrm{~b}$. Formation of CV chondrules by recycling of amoeboid olivine aggregate-like precursors. Geochimica et Cosmochimica Acta 247:121-141.

Marrocchi Y., and Piani L. 2019. The tumultuous childhood of the Solar System. Nature Astronomy 889-890.

Marrocchi Y. et al. 2020. The Piancaldoli meteorite: A forgotten primitive LL3.10 ordinary chondrite. Meteoritics \& Planetary Science 55:maps.13552.

Marrocchi Y., Avice G., and Barrat J.-A. 2021. The Tarda Meteorite: A Window into the Formation of D-type Asteroids. The Astrophysical Journal Letters 913:L9.

Marsset M. et al. 2020. The violent collisional history of aqueously evolved (2) Pallas. Nature Astronomy 4:569-576.

Masiero J. R., Grav T., Mainzer A. K., Nugent C. R., Bauer J. M., Stevenson R., and Sonnett S. 2014. Main-belt asteroids with wise /neowise: near-infrared albedos. The Astrophysical Journal 791:121.

McCord T. B. et al. 2012. Dark material on Vesta from the infall of carbonaceous volatilerich material. Nature 491:83-86.

McSween H. Y., Mittlefehldt D. W., Beck A. W., Mayne R. G., and McCoy T. J. 2011. HED Meteorites and Their Relationship to the Geology of Vesta and the Dawn Mission. Space Science Reviews 163:141-174.

Michel P. 2001. Collisions and Gravitational Reaccumulation: Forming Asteroid Families and Satellites. Science 294:1696-1700. 
Michel P., and Richardson D. C. 2013. Collision and gravitational reaccumulation: Possible formation mechanism of the asteroid Itokawa. Astronomy \& Astrophysics 554:L1.

Michel P. et al. 2020. Collisional formation of top-shaped asteroids and implications for the origins of Ryugu and Bennu. Nature Communications 11:2655.

Minton D. A., and Malhotra R. 2009. A record of planet migration in the main asteroid belt. Nature 457:1109-1111.

Morbidelli A., Bottke W., Nesvorný D., and Levison H. F. 2009. Asteroids Were Born Big. Icarus 204:558-573.

Morbidelli A., Brasser R., Gomes R., Levison H. F., and Tsiganis K. 2010. Evidence from the asteroid belt for a violent past evolution of jupiter's orbit. The Astronomical Journal 140:1391-1401.

Morbidelli A., Bitsch B., Crida A., Gounelle M., Guillot T., Jacobson S., Johansen A., Lambrechts M., and Lega E. 2016. Fossilized condensation lines in the Solar System protoplanetary disk. Icarus 267:368-376.

Morbidelli A., and Raymond S. N. 2016. Challenges in planet formation: Challenges in planet formation. Journal of Geophysical Research: Planets 121:1962-1980.

Morlok A., Bischoff A., Stephan T., Floss C., Zinner E., and Jessberger E. K. 2006. Brecciation and chemical heterogeneities of CI chondrites. Geochimica et Cosmochimica Acta 70:5371-5394.

Nesvorný D., Broz M., and Carruba V. 2015. Identification and Dynamical Properties of Asteroids IV, Patrick Michel, Francesca E. DeMeo, and William F. Bottke (eds.), University of Arizona Press, Tucson, pp. 297-321.

O'Brien D. P., and Sykes M. V. 2011. The origin and evolution of the asteroid beltImplications for Vesta and Ceres. Space science reviews 163:41-61.

Okada T. et al. 2020. Highly porous nature of a primitive asteroid revealed by thermal imaging. Nature 579:518-522.

Pape J., Mezger K., Bouvier A.-S., and Baumgartner L. P. 2019. Time and duration of chondrule formation: Constraints from ${ }^{26} \mathrm{Al}-{ }^{26} \mathrm{Mg}$ ages of individual chondrules. Geochimica et Cosmochimica Acta 244:416-436.

Patzek M., Bischoff A., Visser R., and John T. 2018. Mineralogy of volatile-rich clasts in brecciated meteorites. Meteoritics \& Planetary Science 43:435-22.

Patzek M., Hoppe P., Bischoff A., Visser R., and John T. 2020. Hydrogen isotopic composition of CI- and CM-like clasts from meteorite breccias - Sampling unknown sources of carbonaceous chondrite materials. Geochimica et Cosmochimica Acta 272:177-197.

Petitat M., Marrocchi Y., McKeegan K. D., Mostefaoui S., Meibom A., Zolensky M. E., and Gounelle M. 2011. ${ }^{53} \mathrm{Mn}^{-53} \mathrm{Cr}$ ages of Kaidun carbonates. Meteoritics \& Planetary Science 46:275-283. 
Piani L., Marrocchi Y., Libourel G., and Tissandier L. 2016. Magmatic sulfides in the porphyritic chondrules of EH enstatite chondrites. Geochimica et Cosmochimica Acta 195:84-99.

Piani L., Marrocchi Y., Rigaudier T., Vacher L. G., Thomassin D., and Marty B. 2020. Earth's water may have been inherited from material similar to enstatite chondrite meteorites. Science 369:1110-113.

Piani L., Marrocchi Y., Vacher L. G., Yurimoto H., and Bizzarro M. 2021. Origin of hydrogen isotopic variations in chondritic water and organics. Earth and Planetary Science Letters 567:117008.

Raymond S.N, Mandell A.M., Sigrudsson S. 2006. Exotic Earths: Forming Habitable Worlds with Giant Planet Migration. Science 313:1413-1416.

Raymond S. N., O’Brien D. P., Morbidelli A., and Kaib N. A. 2009. Building the terrestrial planets: Constrained accretion in the inner solar system. Icarus 203:644-662.

Raymond S. N., and Izidoro A. 2017a. Origin of water in the inner Solar System: Planetesimals scattered inward during Jupiter and Saturn's rapid gas accretion. Icarus 297:134-148.

Raymond S. N., and Izidoro A. 2017b. The empty primordial asteroid belt. Science Advances 3:e1701138.

Raymond S. N., and Morbidelli A. 2020. Planet formation: key mechanisms and global models. arXiv:2002.05756.

Rubin A. E. 1983. The Adhi Kot breccia and implications for the origin of chondrules and silica-rich clasts in enstatite chondrites. Earth and Planetary Science Letters 64:201212.

Rubin A. E., and Bottke W. F. 2009. On the origin of shocked and unshocked CM clasts in Hchondrite regolith breccias. Meteoritics \& Planetary Science 44:701-724.

Rubin A. E. 2012. Collisional facilitation of aqueous alteration of CM and CV carbonaceous chondrites. Geochimica et Cosmochimica Acta 90:181-194.

Rubin A. E. 2015. Impact features of enstatite-rich meteorites. Geochemistry 75:1-28.

Safronov V. S., and Zvjagina E. V. 1969. Relative sizes of the largest bodies during the accumulation of planets. Icarus 10:109-115.

Sahijpal S., Soni P., and Gupta G. 2007. Numerical simulations of the differentiation of accreting planetesimals with ${ }^{26} \mathrm{Al}$ and ${ }^{60} \mathrm{Fe}$ as the heat sources. Meteoritics \& Planetary Science 42:1529-1548.

Schenk P. et al. 2012. The Geologically Recent Giant Impact Basins at Vesta's South Pole. Science 336:694-697. 
Schlüter J., Schultz L., Thiedig F., Al-Mahdi B.O., and Abu Aghreb A.E. 2002. The Dar al Gani meteorite field (Libyan Sahara): Geological setting, pairing of meteorites, and recovery density. Meteoritics \& Planetary Science. 37:1070-1093.

Sharp T.G., and DeCarli P.S. 2006. Shock Effects in Meteorites. In Meteorites and the early solar system II, edited by Lauretta D. S. and McSween H. Y. Tucson, Arizona: The University of Arizona Press. pp. 653-677.

Stöffler, D., Hamann, C., \& Metzler, K. 2018. Shock metamorphism of planetary silicate rocks and sediments: Proposal for an updated classification system. Meteoritics \& Planetary Science 53:5-49.

Stooke P.J. 1996. The Surface of Asteroid 951 Gaspra. Earth, Moon and Planets 75:53-75.

Sugita S. et al. 2019. The geomorphology, color, and thermal properties of Ryugu: Implications for parent-body processes. Science 364:252.

Suttle, M. D., King, A. J., Schofield, P. F., Bates, H., \& Russell, S. S. 2021. The aqueous alteration of CM chondrites, a review. Geochimica et Cosmochimica Acta 299:219256.

Tatsumi E. et al. 2021. Collisional history of Ryugu's parent body from bright surface boulders. Nature astronomy 5:39-45.

Terada K. and Bischoff A. 2009. Asteroidal granite-like magmatism $4.53 \mathrm{Gyr}$ ago. The Astrophysical Journal 699, L68-L71.

Thomas P. C., Binzel R. P., Gaffey M. J., Storrs A. D., Wells E. N., and Zellner B. H. 1997. Impact Excavation on Asteroid 4 Vesta: Hubble Space Telescope Results. Science 277:1492-1495.

Tsiganis K., Gomes R., Morbidelli A., and Levison H. F. 2005. Origin of the orbital architecture of the giant planets of the Solar System. Nature 435:459-461.

Tsirvoulis G., Morbidelli A., Delbo M., and Tsiganis K. 2018. Reconstructing the size distribution of the primordial Main Belt. Icarus 304:14-23.

Turner S., McGee L., Humayun M., Creech J., and Zanda B. 2021. Carbonaceous chondrite meteorites experienced fluid flow within the past million years. Science 371:164-167.

Turrini D., Svetsov V., Consolmagno G., Sirono S., and Pirani S. 2016. Olivine on Vesta as exogenous contaminants brought by impacts: Constraints from modeling Vesta's collisional history and from impact simulations. Icarus 280:328-339.

Vacher L. G., Marrocchi Y., Villeneuve J., Verdier-Paoletti M. J., and Gounelle M. 2017. Petrographic and $\mathrm{C} \& \mathrm{O}$ isotopic characteristics of the earliest stages of aqueous alteration of CM chondrites. Geochimica et Cosmochimica Acta 213:271-290.

Vacher L. G., Marrocchi Y., Villeneuve J., Verdier-Paoletti M. J., and Gounelle M. 2018. Collisional and alteration history of the $\mathrm{CM}$ parent body. Geochimica et Cosmochimica Acta 239:213-234. 
Vacher L. G., Truche L., Faure F., Tissandier L., Mosser-Ruck R., and Marrocchi Y. 2019a. Deciphering the conditions of tochilinite and cronstedtite formation in CM chondrites from low temperature hydrothermal experiments. Meteoritics \& Planetary Science. 54:1870-1889.

Vacher L. G., Piralla M., Gounelle M., Bizzarro M., and Marrocchi Y. 2019b. Thermal Evolution of Hydrated Asteroids Inferred from Oxygen Isotopes. The Astrophysical Journal Letters 882:0-0.

Vacher L. G., Piani L., Rigaudier T., Thomassin D., Florin G., Piralla M., and Marrocchi Y. 2020. Hydrogen in chondrites: Influence of parent body alteration and atmospheric contamination on primordial components. Geochimica et Cosmochimica Acta 281:5366.

van Drongelen K. D., Rumble III D. and Tait K. T. 2016. Petrology and oxygen isotopic compositions of clasts in HED polymict breccia NWA 5232. Meteoritics \& Planetary Science 51:1184-1200.

van Schmus W.R., and Wood J.A. 1967. A chemical-petrologic classification for the chondritic meteorites. Geochimica et Cosmochimica Acta 31:747-765.

Verdier-Paoletti M. J., Marrocchi Y., Vacher L. G., Gattacceca J., Gurenko A., Sonzogni C., and Gounelle M. 2019. Testing the genetic relationship between fluid alteration and brecciation in CMchondrites. Meteoritics \& Planetary Science 54:395-18.

Veverka J., Belton M., Klaasen K., and Chapman C. 1994. Galileo's Encounter with 951 Gaspra: Overview. Icarus 107:2-17.

Villeneuve J., Chaussidon M., and Libourel G. 2009. Homogeneous Distribution of ${ }^{26} \mathrm{Al}$ in the Solar System from the Mg Isotopic Composition of Chondrules. Science 325:985988.

Walsh K. J., Morbidelli A., Raymond S. N., O’Brien D. P., and Mandell A. M. 2011. A low mass for Mars from Jupiter's early gas-driven migration. Nature 475:206-209.

Walsh K. J. et al. 2019. Craters, boulders and regolith of (101955) Bennu indicative of an old and dynamic surface. Nature Geoscience 12:242-246.

Wetherill G. W. 1990. Formation of the Earth. Annual Review of Earth and Planetary Sciences 18:205-256.

Wurm G., Paraskov G., and Krauss O. 2005. Growth of planetesimals by impacts at $\sim 25 \mathrm{~m} / \mathrm{s}$. Icarus 178:253-263.

Zolensky M. E., Ivanov A. V., Yang S. V., Mittlefehldt D. W., and Ohsumi K. 1996. The Kaidun meteorite: Mineralogy of an unusual CM1 lithology. Meteoritics \& Planetary Science 31:484-493.

Zolensky M., and Ivanov A. 2003. The Kaidun Microbreccia Meteorite: A Harvest from the Inner and Outer Asteroid Belt. Chemie der Erde - Geochemistry 63:185-246. 

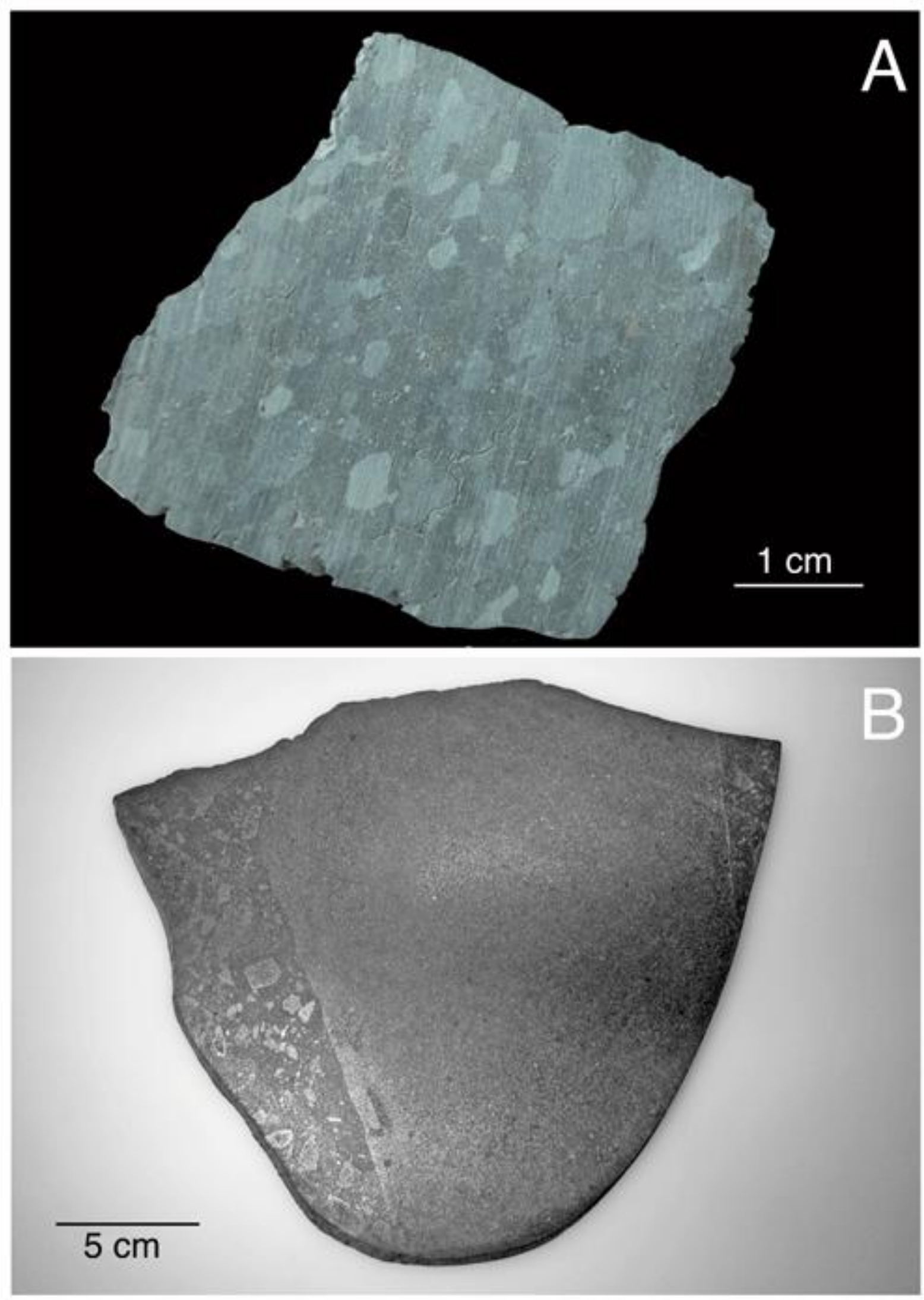

916

917

Fig. 1

918 


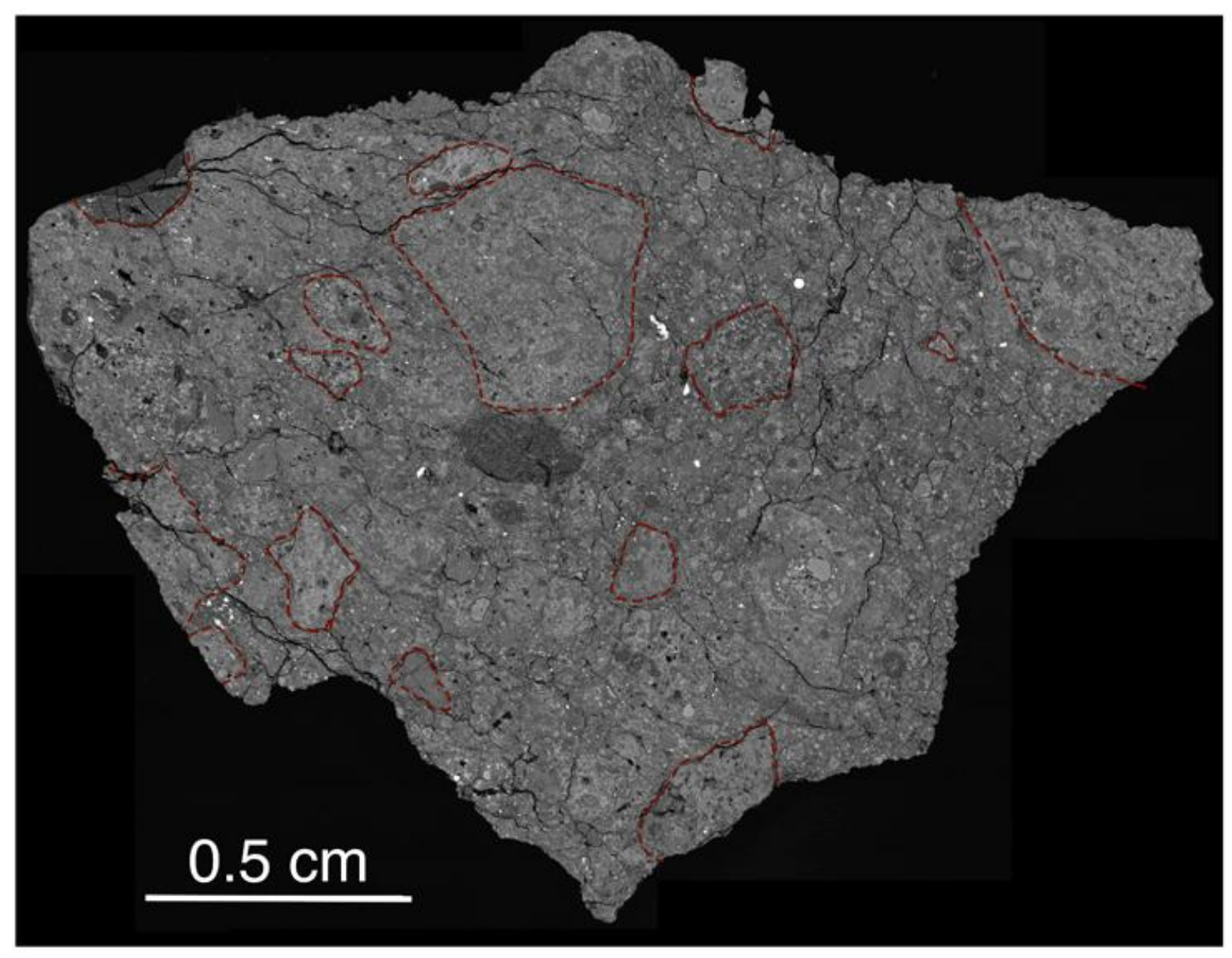

919

920

Fig. 2 


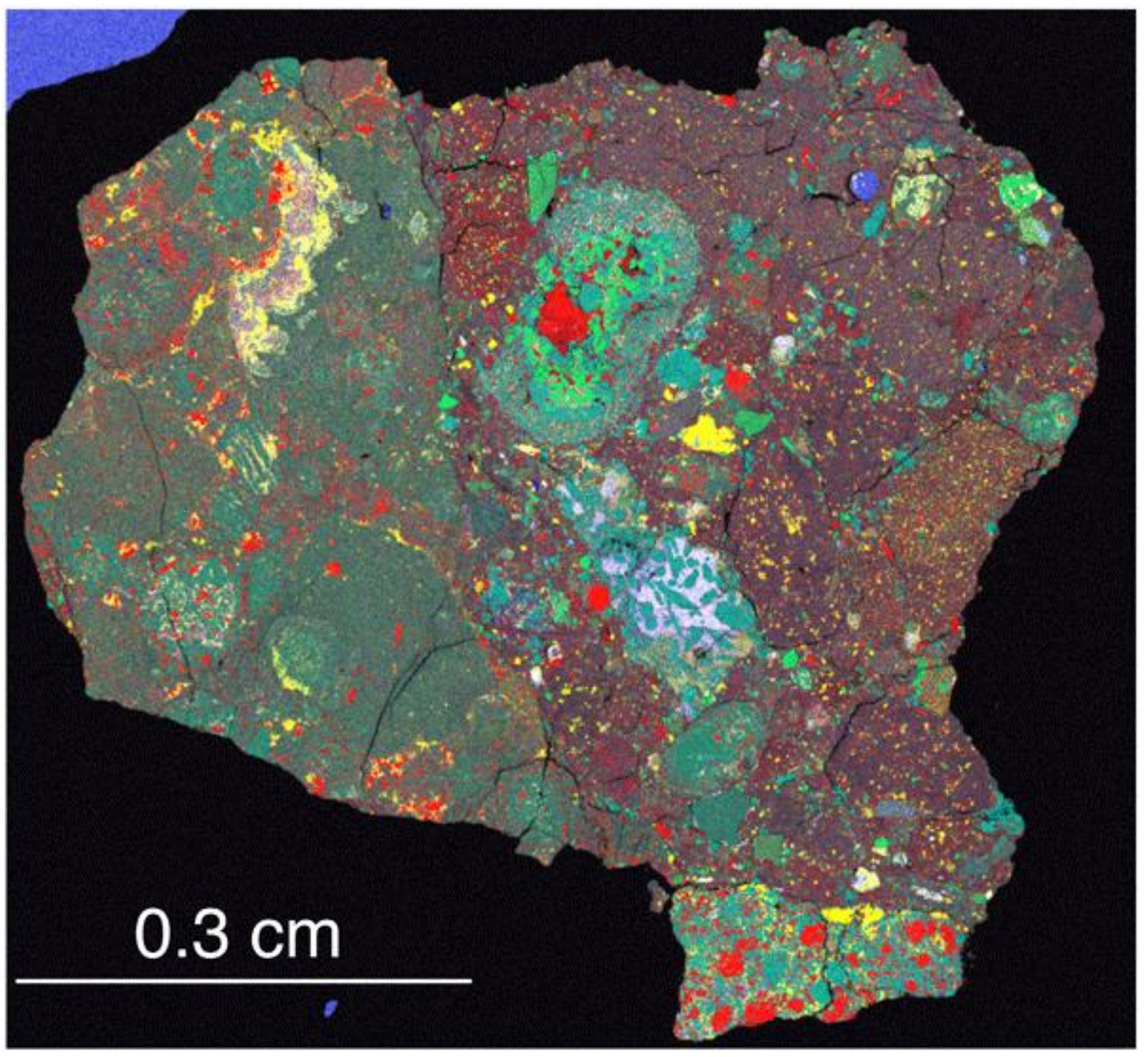

Fig. 3 


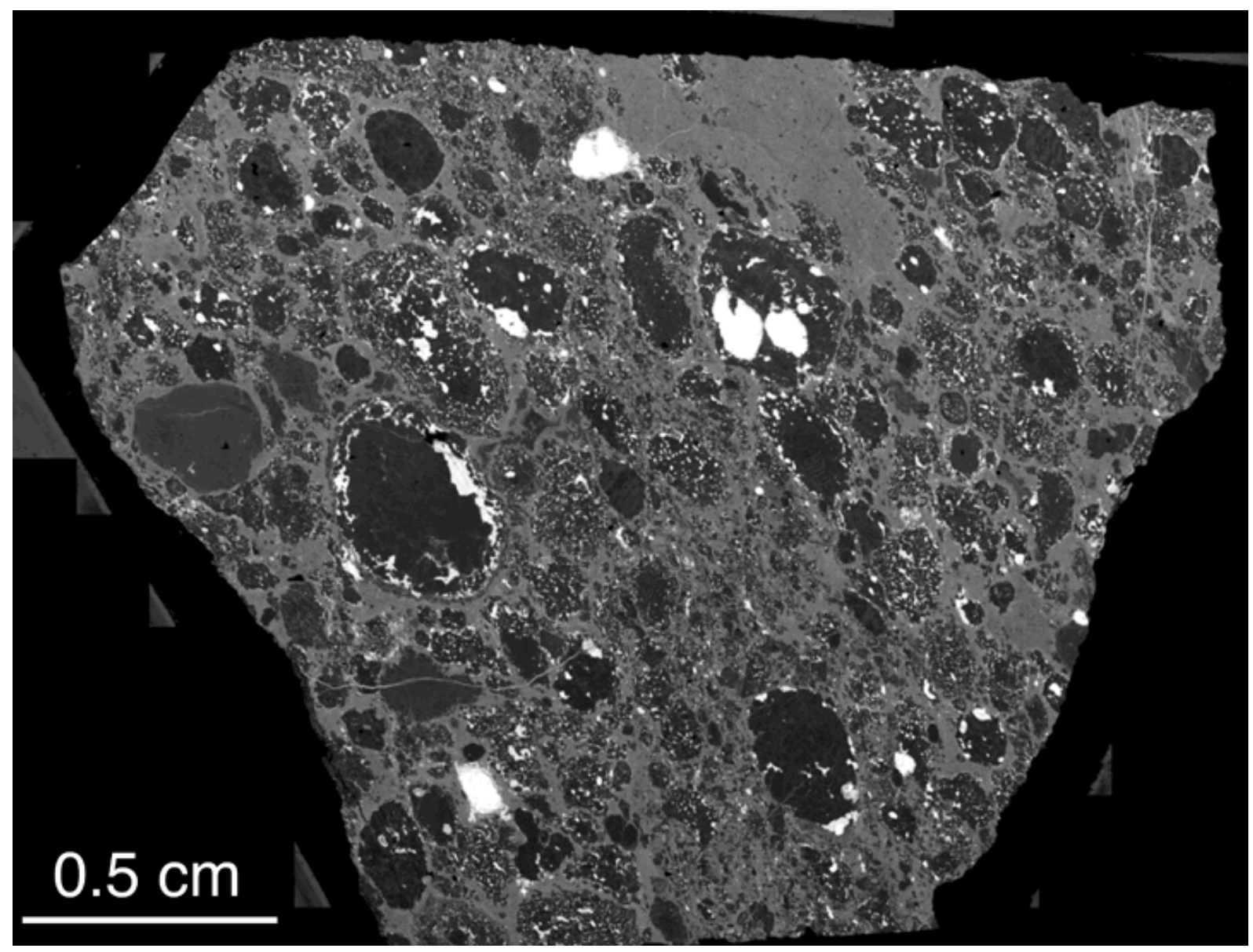

923

924

Fig. 4

925 

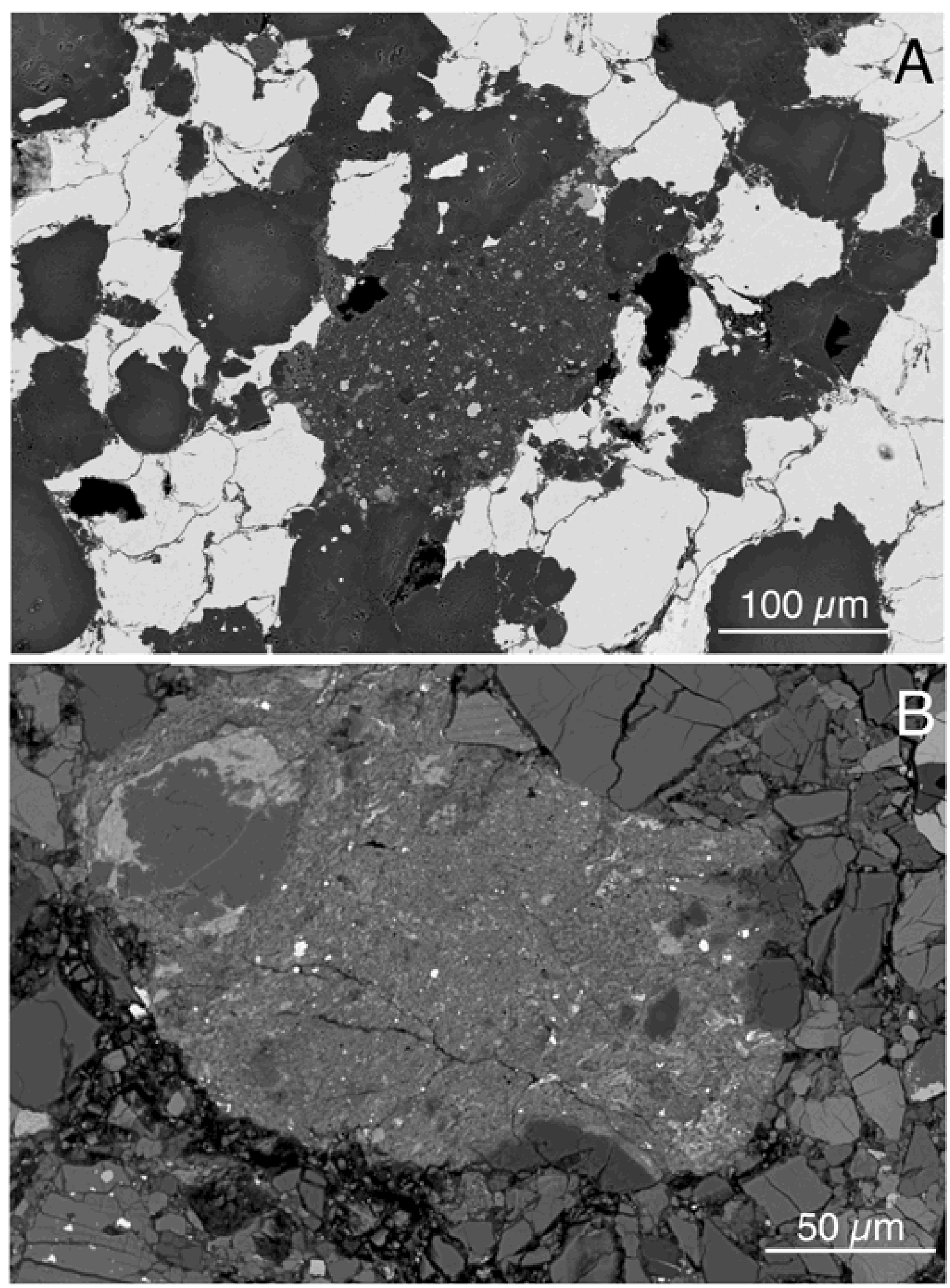

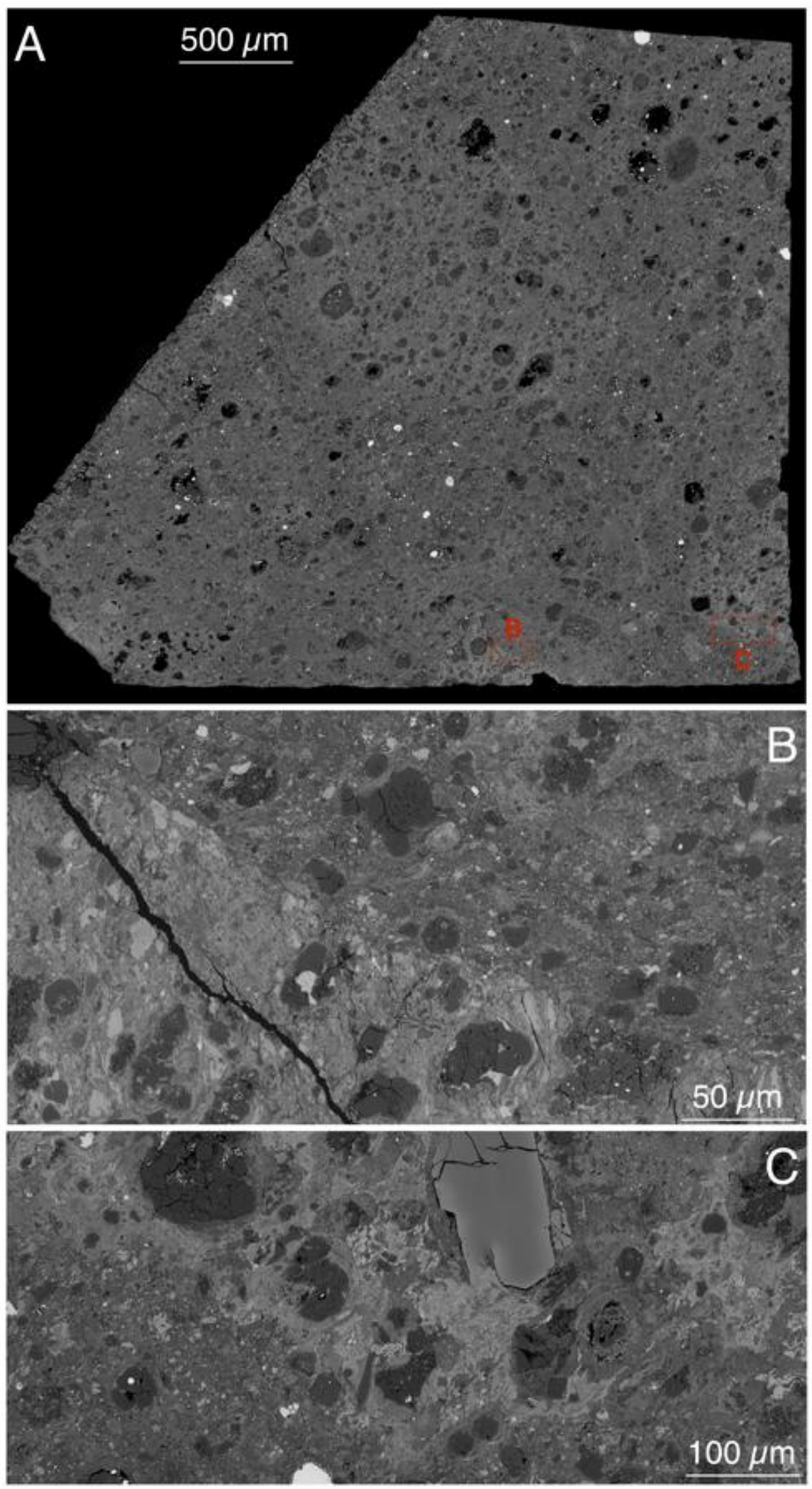

Fig. 6 


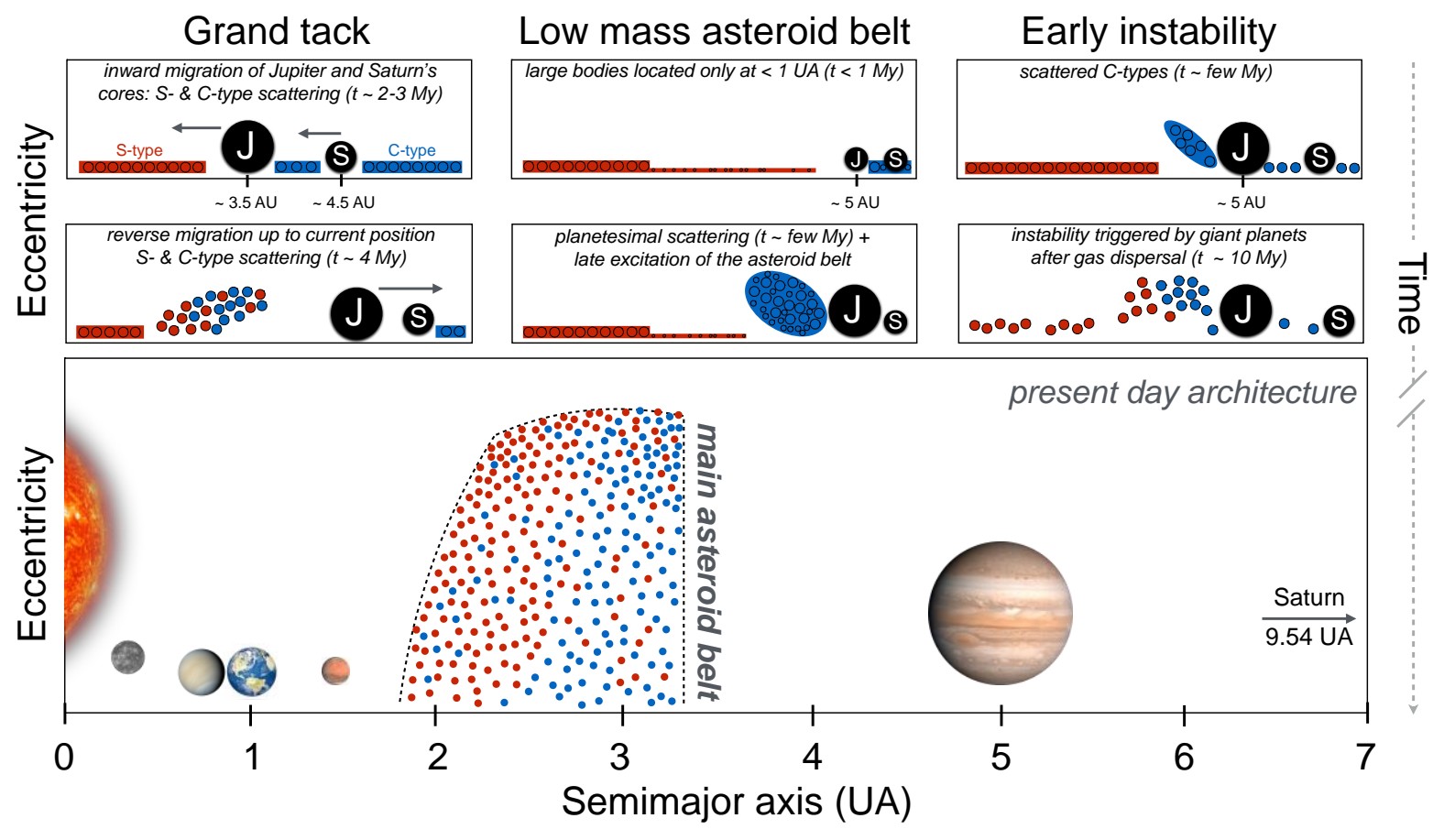

Fig. 7

934

935

936 